\title{
Les outils de la conception dynamique des ponts
}

\author{
The tools for bridge aerodynamical design
}

par C. Crémona

Laboratoire Central des Ponts et Chaussées

The wind capacity to damage and further more to destroy civil engineering structures, has been known since the ancient times. During this century, the well documented destruction of the Tacoma Narrows Bridge in 1940 was a central event for clearing and defining the needs in methods and techniques to design and dimension bridges able to resist to wind. This paper reviews the experimental and numerical tools which are available for forecasting wind effects. It also introduces the new features provided by computational fluid dynamics.

\section{I 口 UN PEU D'HISTOIRE...}

\subsection{Les tournants majeurs dans la conception aérody- namique des ponts}

Comprendre le comportement des ponts sous l'action du vent n'est pas une préoccupation récente. Les progrès dans la construction des ponts suspendus ont toujours constitué un combat continu contre l'action du vent. La plupart des grands ponts suspendus construits au $\mathrm{XIX}^{\mathrm{e}}$ ont d'ailleurs été pour la plupart détruits ou sévèrement endommagés par le vent. En termes de progrès dans l'analyse de l'interaction "vent-pont ", trois événements majeurs peuvent être considérés comme des tournants dans l'histoire de la conception aérodynamique des grands ponts. Le premier est l'effondre-

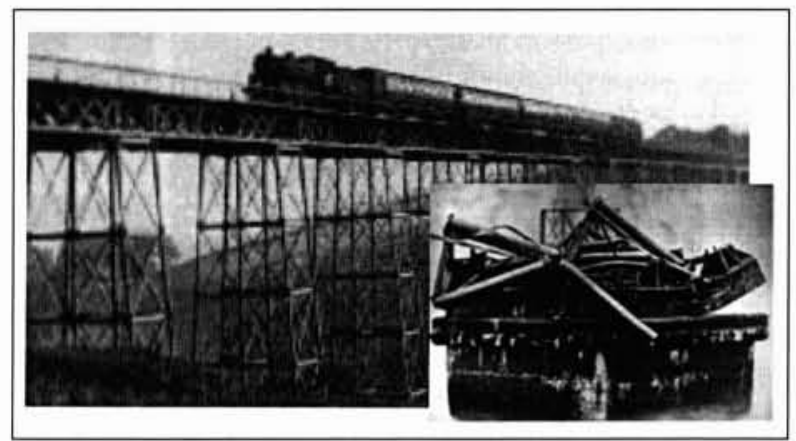

1. Vue du Firth of Tay Bridge ment du Firth of Tay Bridge en 1879. Le Tay Bridge était le record mondial de l'époque avec 84 travées. La portion centrale de l'ouvrage était constituée de 13 travées de $60 \mathrm{~m}$ de longueur à une hauteur de $27 \mathrm{~m}$ au-dessus de la rivière Tay. Ce fut ce tronçon qui s'écroula par grand vent, causant la mort de 80 personnes dans un train. Cet incident, dû à la sous-estimation des effets statiques du vent, provoqua une forte sensibilisation des ingénieurs pour une réelle prise en compte de l'action du vent.

Le second événement est le fameux effondrement du Tacoma Narrows Bridge en 1940. Ce qui se passa alors est aujourd'hui bien connu. L'ouvrage était calculé pour résister aux effets statiques du vent pour des vitesses deux fois supérieures à celle au moment de l'accident. Mais ici, ce n'était pas la mauvaise prise en compte des effets statiques qui était à l'origine de l'effondrement, mais plutôt les effets dynamiques induits par le vent.

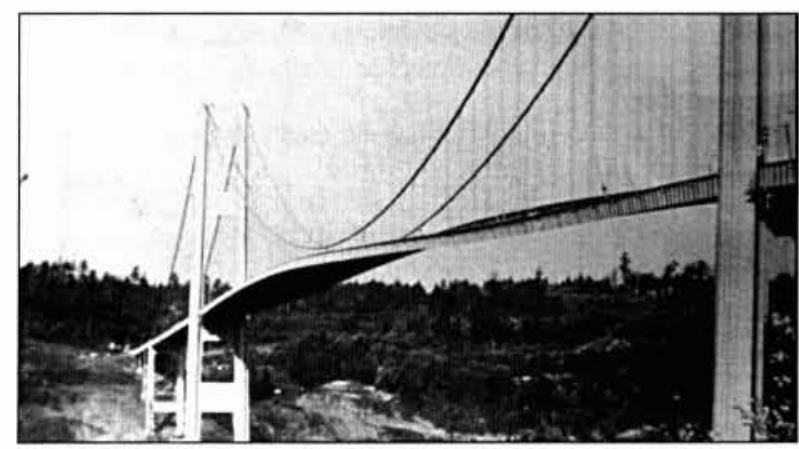

2. Effondrement du Tacoma Narrows Bridge. 
Le troisième tournant dans la conception aérodynamique des ponts est l'utilisation de données climatologiques. A.G. Davenport a été un pionnier dans le domaine en ramenant une quantité considérable d'informations climatologiques à un ensemble de règles et de spécifications pour simuler en soufflerie la couche limite atmosphérique.

A la suite de l'incident du Tacoma Bridge, une contribution importante fut apportée par les ingénieurs de l'aéronautique en recourant à des essais en soufflerie conventionnelle. Les travaux de Davenport sur la simulation du vent naturel en soufflerie fut d'un apport aussi considérable pour la compréhension et l'analyse de la réponse des ponts sous l'action du vent. La deuxième moitié de ce siècle a été l'objet d'un grand nombre d'études aérodynamiques de ponts à travers le monde.

\subsection{Les outils de la conception aérodynamique des ponts}

Le développement d'outils pour la conception des ponts de grande portée a commencé avec l'expertise de l'effondrement du Tacoma Bridge et les études du second Tacoma Bridge. Les investigations de F.B. Farquharson à l'Université de Washington [1] commencèrent avec le développement de procédures d'essais en soufflerie sur des modèles réduits complets de pont. Plus tard, Farquharson utilisa des tronçons (section models) pour étudier la qualité aérodynamique des tabliers de pont. C'est aujourd'hui l'approche la plus utilisée (cf $\S 2.2$ ).

Les essais sur modèle aéroélastique complet ont connu récemment un regain d'attention avec l'accélération des records mondiaux de portée. A la différence des travaux de Farquharson qui étaient réalisés en écoulement laminaire, les essais actuels sont menés en soufflerie à couche limite. On doit cependant à Davenport [2] d'avoir revisité les modèles réduits tridimensionnels en introduisant le concept de modèle à fils tendus (taut-strip model). La méthode partage de nombreux avantages avec les modèles réduits complets mais reste cependant de mise en œuvre plus simple (cf $\$ 2.2$ ). Ces méthodes sont cependant onéreuses et sont la plupart du temps mises en ouvre en phase finale de conception.

Le développement d'outils analytiques pour l'étude des performances aérodynamiques des ponts a également débuté avec l'incident du Tacoma Bridge et en parallèle avec les investigations expérimentales. Les premiers travaux ont concerné l'étude de la stabilité par rapport aux phénomènes aéroélastiques. Bleich [3] et Rocard [4] ont ainsi adapté la théorie des ailes d'avion de Theodorsen [5] au cas des ponts, notamment pour calculer les vitesses critiques de flottement. Mais ce sont surtout les travaux de Scanlan [6] qui ont permis d'établir des modèles décrivant les forces aéroélastiques pour les tabliers de pont. La différence fondamentale de ces modèles par rapport à ceux de l'aéronautique vient du fait qu'ils font largement appel à des paramètres expérimentaux de la section concernée.

Plus tardivement, dans les années soixante, on commença à étudier la réponse dynamique de la structure sous la turbulence du vent. La nature aléatoire du vent au voisinage du sol fait de la théorie des vibrations aléatoires l'outil logique pour appréhender la réponse turbulente des structures de génie civil. Les premières applications de cette théorie à l'étude des ponts souples sont dues à Davenport [7]. L'approche consiste à analyser l'effet des turbulences (caractérisées par des fonctions de densité spectrale) comme un chargement stochastique qui met en vibration la structure conformément à un phénomène de résonance. Habituellement, la structure est considérée comme ayant un amortissement de Rayleigh. Cette dernière hypothèse permet de découpler les équations du mouvement (en utilisant la base des modes propres non amortis) en un ensemble d'équations modales indépendantes. Il est alors possible d'évaluer séparément la densité spectrale de puissance et l'écart-type de la réponse de chaque mode.

Ces deux problématiques (l'étude de la stabilité et l'évaluation de la réponse à la turbulence) furent développées indépendamment et l'interaction entre les deux phénomènes fut longtemps négligée. Or, dans la plupart des structures, les équations modales sont couplées par les termes d'amortissement et, dans le cas des grands ponts, par des termes aéroélastiques. D'un point de vue général, les effets aéroélastiques peuvent être vus comme des termes correctifs à l'amortissement et la rigidité du pont. Durant les vingt dernières années, de nombreuses méthodes ont été proposées pour considérer de façon plus réaliste les effets de l'interaction fluide-structure dans la réponse à la turbulence, soit dans le domaine de la fréquence [8], [9], [10], soit dans le domaine du temps [11], [10].

L'analyse du comportement mécanique des structures a largement bénéficié du développement de la méthode des éléments finis. Ainsi, il est aujourd'hui très rare de recourir à des essais en laboratoire durant les études de conception et les méthodes analytiques "à la main " ne sont utilisées que dans les études préliminaires. Des développements similaires peuvent s'envisager pour l'évaluation des performances aérodynamiques des éléments de pont. Les codes de calcul de mécanique de fluide et d'interaction fluide-structure reçoivent aujourd'hui une attention particulière [12], [13]. Ils offrent la perspective aux concepteurs de disposer d'un outil pour l'évaluation de plusieurs solutions avant de tester la configuration finale en soufflerie. C'est d'ailleurs l'approche adoptée en aéronautique ou en construction automobile, et c'est sans nul doute une des voies de recherche principales dans le développement de nouveaux outils pour la conception aérodynamique des ponts.

Les sections suivantes présentent succinctement chacun de ces outils et les quelques problèmes rencontrés dans la conception aérodynamique des ponts.

\section{II ÉTUDES EN SOUFFLERIE}

Les proportions du tablier d'un très grand pont - en général suspendu ou à haubans - sont celles d'un long ruban. Il est intuitivement évident qu'une telle structure sera très flexible et particulièrement sujette aux effets du vent. Un problème permanent et récurrent dans la conception de ces ouvrages est de tenir compte de ces effets et de la réponse de la structure qu'ils vont induire afin de produire un "ouvrage satisfaisant » où les efforts, les déplacements et les phénomènes de fatigue restent dans des limites acceptables.

Les structures élancées et flexibles possédant des sections transversales peu ou non profilées, comme les tabliers de pont, les câbles, les pylônes,..., sont particulièrement sensibles aux effets du vent. Ces ouvrages sont généralement dimensionnés pour résister aux charges statiques de vents correspondant à des périodes de retour élevées. Cependant, l'interaction fluide-structure peut engendrer des phénomènes aéroélastiques qui mettent en défaut des méthodologies de calcul basées sur des considérations statiques. Ces phénomènes sont connus sous les noms de divergence en torsion, d'échappement tourbillonnaire, de flottement, de galop, de réponse à la turbulence, ou d'excitation conjointe vent-pluie.

L'étude de ces phénomènes est aujourd'hui menée sur la base d'informations fournies lors d'essais en soufflerie.

\subsection{Les règles de similitude}

Les souffleries aérodynamiques à couche limite constituent un des outils essentiels pour comprendre et évaluer les effets du vent sur les structures de génie civil. Au travers de règles 


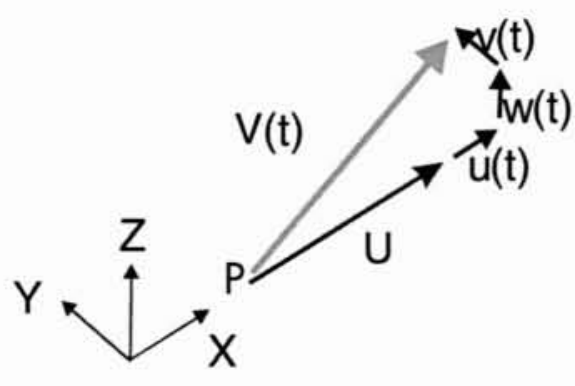

3. Vitesse instantanée du vent.

de similitude, des modèles réduits peuvent être testés pour simuler des conditions à l'échelle 1 .

\subsubsection{Similitude du vent}

La simulation du vent peut être considérée suivant deux manières :

- La simulation des caractéristiques moyennes du vent dans la couche limite turbulente (champ lointain);

- La simulation des caractéristiques du vent à proximité de l'ouvrage largement influencées par les conditions topographiques (champ proche).

L'écoulement en champ lointain est défini par les paramètres suivants (fig. 3 ) :

- Vitesse moyenne du vent dans un repère donné $U(x, y$, $z)$;

- Densité spectrale de puissance des composantes fluctuantes :

- Intensités de turbulence $I_{u}, I_{v}, I_{w}$;

- Echelles de turbulence $L_{u}^{x}, L_{u}{ }^{y}, L_{w}{ }^{y}$;

- Densités spectrales de puissance $S_{u}(n), S_{v}(n), S_{w}(n)$, $S_{u w}(n)$;

- Corrélations spatiales entre composantes $R_{u u}(x, y, z)$, $R_{y, w}(x, y, z), R_{u w w}(x, y, z) \ldots$;

- Température et humidité dans le champ concerné :

- Autres facteurs mineurs comme la force de Coriolis, le gradient de pression,...

Pour la simulation d'un vent naturel en un point précis, les effets de la topographie locale doivent être introduits. Cela est réalisé en reproduisant à échelle réduite le site à proximité de la maquette testée en soufflerie. Une simulation parfaite d'un écoulement turbulent est académique et impraticable. En général, les données à l'échelle 1 sont incomplètes. La vitesse moyenne de l'écoulement coöncide dans la plupart des analyses avec l'axe de la veine. Les facteurs les plus importants à simuler sont :

- $U(y), I_{u}, I_{w}, S_{w}(n)$ et $R_{w w}(y)$ et au niveau du tablier : ces paramètres sont directement reliés à l'action moyenne du vent, et à la réponse dynamique de l'ouvrage dans les trois directions (pompage, tangage et balancement) ;

- $S_{u}(n)$, y compris l'échelle de turbulence longitudinale, est directement reliée au balancement. Ce mode de réponse est sans grand intérêt pour les ponts suspendus en comparaison des deux autres mais peut l'être pour les ponts à haubans à cause du risque de couplage en torsion. Il peut être également relié au pompage et au tangage au travers de sa contribution suivant l'angle d'incidence du vent ;

- $U(z), I_{u}(z)$ et $I_{v}(z)$ sont utiles pour la réponse des pylônes et des câbles.

Une bonne technique de simulation du champ lointain est la soufflerie à couche limite en appliquant des rugosités et des obstacles avec contrôle de la température et de l'humidité. Cette technique est aujourd'hui très répandue bien qu'elle soit à utiliser avec précaution.

\subsubsection{Similarité aéroélastique}

La similarité aéroélastique est effectuée au travers de plusieurs conditions et en fonction de facteurs d'échelle prédéfinis. Les deux principaux facteurs d'échelle sont respectivement :

- l'échelle des longueurs $\lambda_{L}=L_{\text {maquete }} / L_{\text {pont }}$. Son choix est conditionné par la taille et la vitesse de la soufflerie (nombre de Reynolds le plus grand possible sans effet de blocage) ainsi que par sa possibilité, lorsqu'elle est à couche limite, de simuler le vent naturel à différentes échelles. Les échelles habituelles sont donc de l'ordre du 1/30 au 1/100 pour les essais sur tronçon partiel de tablier de pont, et du $1 / 100$ au $1 / 500$ lorsque l'on considère une maquette du pont entier ;

- l'échelle des vitesses $\lambda_{U}=U_{\text {soufferie }} / U_{\text {site }}$. Le choix est également arbitraire mais de faibles vitesses sont à éviter si l'on souhaite conserver un nombre de Reynolds suffisamment grand et un bon rapport signal/bruit de l'instrumentation de mesure.

Ces échelles sont choisies arbitrairement ; cependant, la vérification de certaines règles de similitudes oblige qu'elles vérifient certaines relations.

\section{Similitude de Froude}

Le nombre de Froude représente le rapport entre les forces d'inertie et la force de gravité. Ce nombre est particulièrement important pour les ponts suspendus où l'action de la gravité est importante. La condition de Froude impose :

$$
F r_{\text {maquette }}=F r_{\text {pont }} \Rightarrow\left(\frac{U}{\sqrt{L \cdot g}}\right)_{\text {maquette }}=\left(\frac{U}{\sqrt{L \cdot g}}\right)_{\text {pont }} \Rightarrow \lambda_{U}=\sqrt{\lambda_{L}}
$$

\section{Similitude de la fréquence réduite}

La fréquence réduite est définie par le rapport $K=(n L) / U$. La condition de similitude sur la fréquence réduite implique alors :

$$
K_{\text {maquente }}=K_{\text {pont }} \Rightarrow\left(\frac{n L}{U}\right)_{\text {maquette }}=\left(\frac{n L}{U}\right)_{\text {pont }} \Rightarrow \lambda_{n}=\lambda_{U} / \lambda_{L}
$$

\section{Similitude de Reynolds}

Le nombre de Reynolds est le rapport entre les forces d'inertie du fluide et les forces visqueuses. L'égalité des nombres de Reynolds entre pont réel et maquette n'est pas possible. L'air étant le fluide commun entre soufflerie et vraie grandeur, le respect de la condition de Reynolds implique une échelle des vitesses inverse de l'échelle géométrique, ce qui conduirait à des régimes d'écoulement supersoniques pour les échelles de simulation habituellement utilisées. Cependant, l'invalidité de la condition de Reynolds doit être examinée avec attention pour une interprétation correcte des résultats. En particulier, dans le cas d'obstacles mal profilés aux arêtes vives, les points de séparation sont fixes et l'écoulement est supposé être peu sensible au nombre de Reynolds. C'est le cas des tabliers de pont. Cependant, le recollement est, lui, dépendant du nombre de Reynolds et il peut en résulter une réduction de la traînée et une augmentation du nombre de Strouhal.

\section{Similitude d'amortissement}

La relation de similitude d'amortissement consiste à conserver les mêmes taux d'amortissement entre pont réel et maquette. Cependant, ce taux est généralement inconnu en phase de conception, ou très mal estimé pour les ouvrages existants. Des valeurs forfaitaires sont alors utilisées.

\subsection{Les types d'essais en soufflerie}

Pour les essais sur les ponts, on distingue généralement deux types de maquettes, un modèle partiel représentant un tron- 


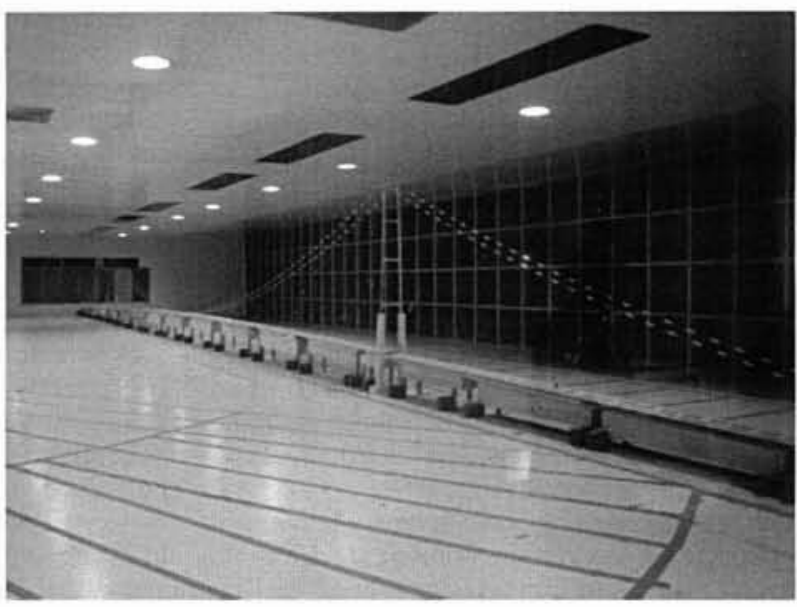

4. Exemple de modèle complet (projet de pont au Japon).

çon de tablier de pont, et un modèle qui représente l'ensemble du pont (modèle complet) ou une partie de l'ouvrage (modèle à fils tendus).

\subsubsection{Les essais sur modèle aéroélastique complet}

En plus d'être géométriquement similaire à l'ouvrage réel (fig. 4), de tels modèles doivent satisfaire des conditions de similarité de répartition des masses, de fréquence réduite, d'amortissement mécanique et de modes propres de vibrations. La construction d'un modèle réduit complet d'un ouvrage est difficile d'élaboration et est surtout très coûteux. Les dimensions de ces modèles sont en général au $1 / 300^{\circ}$ bien que des échelles au $1 / 100^{\mathrm{e}}$ aient été utilisées dans certains cas.

\subsubsection{Les essais sur modèle à fils tendus}

Le modèle à fils tendus permet de reproduire le caractère tridimensionnel des modes de vibrations sans recourir à la modélisation des pylônes et des haubans. Avec cette technique, la maquette n'est pas dimensionnée en fonction de la taille du pont, mais des dimensions de la turbulence envisageable dans la veine (fig.5). Dans la plupart des cas, les échelles de longueur et vitesse sont choisies indépendamment l'une de l'autre. Par conséquent, la condition de similitude de Froude peut ne pas être vérifiée. Ceci n'est pas

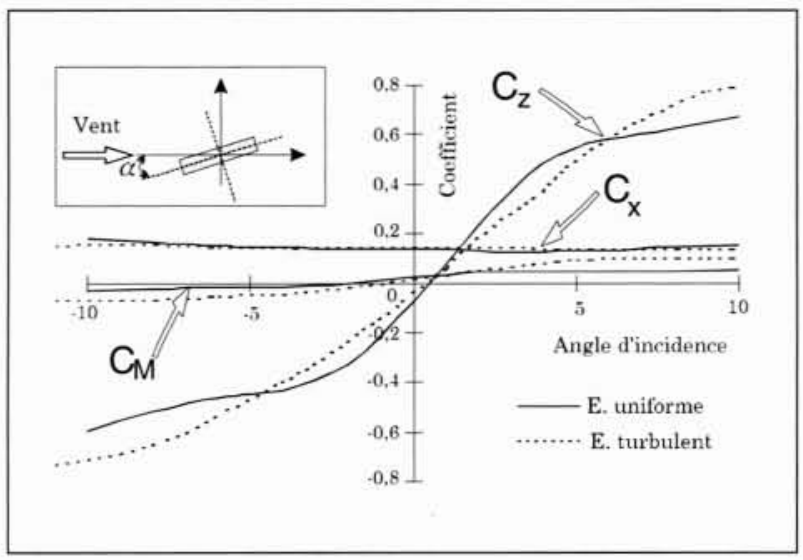

6. Coefficients aérodynamiques du pont Vasco de Gama.

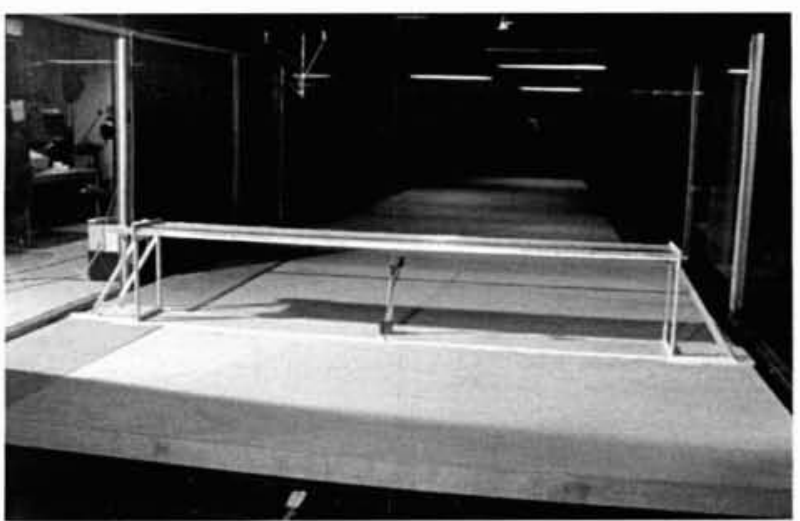

5. Modèle à fils tendus du pont Vasco de Gama (photo CSTB).

gênant si la force de gravité joue un rôle mineur. C'est le cas des ponts à haubans.

\subsubsection{Les essais sur tronçons}

L'étude en soufflerie est indispensable pour qualifier la qualité aérodynamique des éléments du pont et notamment de son tablier, partie sensible de l'ouvrage où naît une grande partie des forces aérodynamiques. La méthode expérimentale la plus simple et la moins onéreuse est d'effectuer des essais sur un tronçon de tablier placé transversalement entre deux plaques d'extrémité, face au vent incident, dans la veine de la soufflerie. Deux types de montage sont utilisés :

- Un montage « raide " qui permet de mesurer les forces aérodynamiques de portance, de traînée et de torsion, et donc les coefficients correspondants nécessaires au calcul des effets statiques et à ceux de la turbulence (fig. 6) ;

- Un montage « souple » où différents degrés de liberté sont simulés par des actionneurs. Ce montage permet d'appréhender la sensibilité du tablier au détachement tourbillonnaire, et de déterminer les effets instationnaires ou aéroélastiques s'exerçant sur le tablier (fig. 7). Deux méthodes d'études sont envisageables: oscillations libres ou oscillations forcées. Dans la première méthode d'analyse, les taux d'amortissement et les décalages fréquentiels permettent d'extraire les coefficients instationnaires correspondants. La seconde méthode impose fréquence et amplitude de vibration. Les forces sont alors déterminées par intégration des mesures de pression synchrones sur des coupes transversales du tablier, ou par mesure directe après soustraction des forces d'inertie.

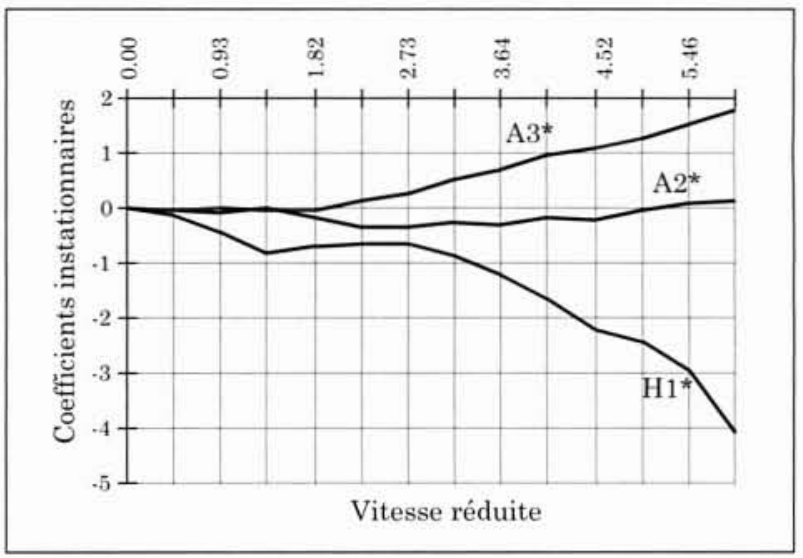

7. Coefficients instationnaires du pont Vasco de Gama. 


\section{III —ÉTUDE NUMÉRIQUE DU COMPOR- TEMENT AU VENT DES PONTS}

Les essais sur tronçons fournissent les informations de base à toute étude analytique menée pour dimensionner un ouvrage. Ces études analytiques peuvent reposer sur deux approches possibles :

- L'approche quasi-stationnaire : les forces instantanées produites par le vent turbulent sur un obstacle sont égales à celles qui se produiraient sur celui-ci dans un écoulement permanent avec la même vitesse et le même angle d'incidence ;

- L'approche aéroélastique : l'interaction fluide-structure est effectivement prise en compte, c'est-à-dire que le mouvement du pont va interagir avec l'écoulement, modifiant ainsi les forces qui s'exercent sur lui.

\subsection{Approche quasi-stationnaire}

Considérons que la direction de la vitesse moyenne du vent fait un angle d'incidence nul (par rapport au trièdre lié à l'obstacle), et que les effets de la composante longitudinale de la turbulence $v(t)$, parallèle à l'axe de ce dernier, soient négligeables :

$$
V(t)=\left\{\begin{array}{c}
U_{\text {ref }} \\
0
\end{array}\right\}+\frac{v^{\prime}(t)}{\left\{\begin{array}{l}
u(t) \\
w(t)
\end{array}\right\}}
$$

Supposons que cet obstacle est en oscillation. Dans le repère lié à l'obstacle, le module de la vitesse apparente du vent s'exprime au premier ordre par :

$$
\left|V_{\text {app. }}(t)\right|^{2} \approx U_{r e f}^{2}+2 U_{r e f} u(t) \pm 2 U_{r e f} q(t)
$$

où $q(t)$ et $\dot{h}(\mathrm{t})$ et sont respectivement les vitesses d'oscillation horizontale et verticale à l'instant $\mathrm{t}$ (fig. 8). De façon similaire, en négligeant les termes d'ordre supérieur à 2 , l'angle d'incidence apparent du vent s'exprime par :

$$
i_{a p p .}(t)=\frac{w(t)}{U_{r e f}}+\frac{\dot{h}(t)}{U_{r e f}}+\alpha+r B \frac{\dot{\alpha}}{U_{r e f}}
$$

$r$ indique la position du point d'action des forces aérodynamiques par rapport au centre de gravité. Pour une plaque de faible épaisseur, ce point se situe au quart avant de la plaque (fig. 8). En général, pour des tabliers de pont, le point d'action des forces est supposé confondu avec le centre de gravité de la section.

Compte tenu des équations (4) et (5) et en linéarisant les coefficients aérodynamiques autour d'un angle d'incidence nul, les efforts aérodynamiques s'écrivent :

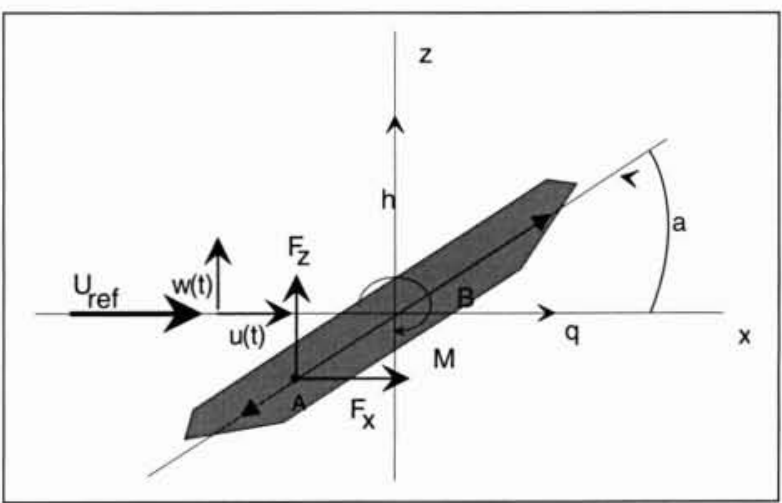

8. Forces aérodynamiques et déplacements en un point courant d'un obstacle.

$$
\begin{gathered}
\frac{F_{j}(t)}{F_{j}^{0}}=C_{j}(0)=\left(1+\frac{2 u(t)}{U_{r e f}}\right)+\frac{d C_{j}}{d i}(0) \frac{w(t)}{U_{r e f}} \\
+\frac{d C_{j}}{d i}(0)\left[r B \frac{\dot{\alpha}(t)}{U_{r e f}}+\frac{\dot{h}(t)}{U_{r e f}}+\alpha(t)\right] \pm C_{j}(0) \frac{2 \dot{q}(t)}{U_{r e f}}
\end{gathered}
$$

où $C_{j}(0)$ et $\frac{d C_{j}}{d i}(0)(j=x, z, M)$ sont les coefficients aérodynamiques et leurs dérivées par rapport à l'angle d'incidence liés au trièdre de l'obstacle, et $B$ la largeur du tablier. $F_{j}^{0}$ est l'effort de référence $\frac{1}{2} \rho B U_{\text {ref }}^{2}$ pour $j=x, z$, et le moment de référence $\frac{1}{2} \rho B^{2} U_{r e f}^{2}$ pour $j=M$. L'approche quasi-stationnaire linéarisée ne présuppose aucune adaptation du fluide au cours du mouvement de l'obstacle. De même, elle ne tient pas compte directement du fait que les fluctuations turbulentes sont constituées d'un train d'onde qui traverse transversalement l'obstacle. Ces limitations de l'approche quasi-stationnaire sont levées en passant à une formulation aéroélastique et en introduisant le concept d'admittance aérodynamique.

\section{- 3.2 Approche aéroélastique}

Les forces ou effets aéroélastiques se développent dans une structure souple lorsque les déplacements de celles-ci et les forces aérodynamiques interagissent. Des phénomènes très complexes ont lieu car la structure en oscillation perturbe l'écoulement du vent, et par conséquent les forces générées sur elle-même.

Le flottement peut être défini comme un phénomène d'oscillations auto-entretenues du tablier du pont qui tire son énergie du vent. Ces oscillations peuvent être soit en torsion, soit en flexion, soit en couplage flexion-torsion. Les oscillations s'amortissent si l'énergie dissipée par la structure est plus grande que celle apportée par le vent; dans le cas contraire, elles augmentent et provoquent l'instabilité. C'est ce type de phénomènes, mal connus à l'époque, qui se trouve à l'origine de l'effondrement spectaculaire du Tacoma Narrows. En aéronautique, le phénomène de flottement des ailes d'avion dans un écoulement stationnaire a fait l'objet de nombreuses études théoriques dès 1925 par Wagner, suivies des travaux de Theodorsen en 1935 [14]. Dans le cas des ailes d'avion, ces problèmes ont été maîtrisés depuis longtemps par le "profilage » de la section. Malheureusement, le fait que les tabliers de pont aient des sections transversales de géométrie "complexe " (non profilée), ne permet pas d'appliquer directement ces théories aux ponts (fig. 9). Pourtant, elles ont servi de " guide " à l'appréhension de ce type de phénomènes dans le cas des ponts.

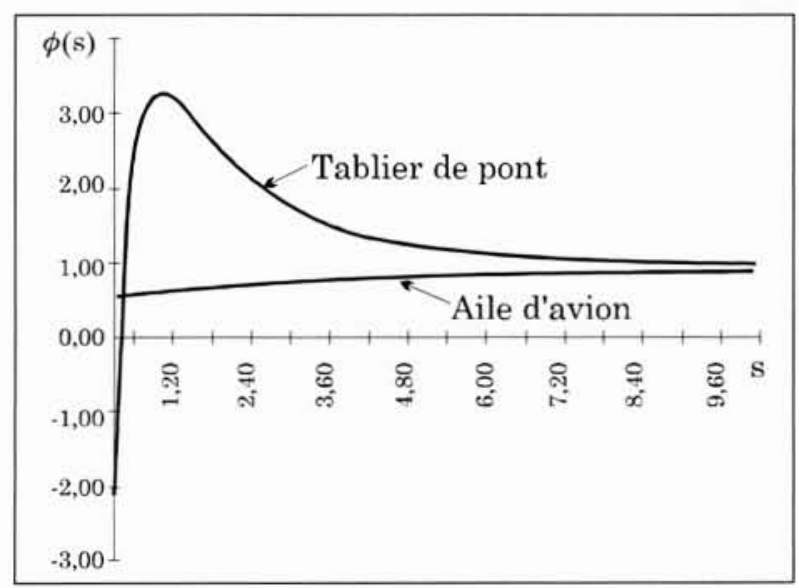

9. Fonctions indicielles (d'après [15]). 
Dans une démarche aéroélastique, la seconde partie de l'équation (6) (relative aux déplacements de l'obstacle) est remplacée par :

- En temporel, par des fonctions indicielles $\Phi_{j}$ qui tiennent compte de l'adaptation du fluide au mouvement $(j=x, z, M)$ :

$$
\begin{gathered}
\frac{F_{j}(t)}{F_{j}^{0}}=\int_{ \pm \infty}^{t} \alpha(\sigma) \Phi_{j \alpha}^{\prime}(t \pm \sigma) d \sigma+\int_{ \pm \infty}^{t} \dot{\alpha}(\sigma) \Phi_{j \alpha}(t \pm \sigma) d \sigma \\
+\int_{ \pm \infty}^{t} q(\sigma) \Phi_{j q}^{\prime}(t \pm \sigma) d \sigma+\int_{ \pm \infty}^{t} \dot{q}(\sigma) \Phi_{j q}(t \pm \sigma) d \sigma \\
+\int_{ \pm \infty}^{t} h(\sigma) \Phi_{j h}^{\prime}(t \pm \sigma) d \sigma+\int_{ \pm \infty}^{t} \dot{h}(\sigma) \Phi_{j i}(t \pm \sigma) d \sigma
\end{gathered}
$$

- En fréquentiel, par les coefficients instationnaires - ou dit de Scanlan - :

$$
\begin{gathered}
\frac{F(w)}{F_{j}^{0}}=\left[K G_{j 1}^{*}(K) \frac{\dot{h}(\omega)}{U_{r e f}}+K G_{j 2}^{*}(K)\left(\frac{B \dot{\alpha}(\omega)}{U_{r e f}}\right)+K^{2} G_{j 3}^{*}(K) \alpha(\omega)\right] \\
\left.+K^{2} G_{j 4}^{*}(K) \frac{h(\omega)}{B}+K G_{j 5}^{*}(K) \frac{\dot{q}(\omega)}{U_{r e f}}+K^{2} G_{j 6}^{*}(K) \frac{q(\omega)}{B}\right]
\end{gathered}
$$

avec $K=\frac{B \omega}{U_{r e f}}$ fréquence réduite. Les fonctions $G_{j l}{ }^{*}(K)$ sont les coefficients instationnaires ou de Scanlan (flutter derivatives). Ils ont en général leur notation propre : $G_{z l}{ }^{*}=H_{i}{ }^{*}$ (pompage), $G_{x l}{ }^{*}=P_{l}^{*}$ (balancement) et $G_{M l}{ }^{*}=A_{i}{ }^{*}$ (tangage) pour $l=1, \ldots, 6$.

Lorsque la fréquence réduite $K$ tend vers 0 , les coefficients instationnaires relatifs à chaque degré de liberté (déplacements et vitesses) doivent tendre vers leurs équivalents introduits par l'approche quasi-stationnaire. Ainsi, en utilisant une approche quasi-stationnaire, Scanlan [16] a établi que les termes du type $K^{2} A_{3}{ }^{*}(K)$ qui apparaissent dans les expressions sont analogues aux dérivées des coefficients aéroélastiques correspondants. On peut alors généraliser ce résultat et monter que, par exemple, $K H_{1}^{*}(K) \underset{k \rightarrow 0}{\longrightarrow} \frac{d C_{z}}{d \alpha}$ et que $K^{2} A_{3}^{*}(K) \underset{k \rightarrow 0}{\longrightarrow} \frac{d C_{M}}{d \alpha}$. Ces comportements asymptotiques peuvent constituer un bon moyen de vérifier (pour un écoulement faiblement turbulent ou laminaire) la bonne adéquation entre des séries de mesures statiques et aéroélastiques.

\subsection{Admittance aérodynamique}

Küssner en 1936 et Sears en 1941 ont permis d'évaluer le rôle de la turbulence du vent sur la réponse, en utilisant la même approche que Wagner. La représentation des effets de la turbulence [14] fait appel à des fonctions indicielles analogues à celle définie par Küssner :

$\frac{F_{j}(t)}{F_{j}^{0}}=C(0) \int_{ \pm \infty}^{t} \frac{2 \mu(\sigma)}{U_{r e f}} \psi_{j u}(t \pm \sigma) d \sigma+\frac{d C_{j}}{d i}(0) \int_{ \pm \infty}^{t} \frac{w(\sigma)}{U_{r e f}} \psi_{j w}(t \pm \sigma)$

où $\Psi_{i l}(j=x, z, M, l=u, w)$ sont les fonctions indicielles relatives â la turbulence. Ces effets peuvent être exprimées dans le domaine de la fréquence au travers de leur densité spectrale de puissance, très utile pour un calcul spectral [10] :

$$
\begin{aligned}
& \frac{S_{j}(w)}{S_{j}^{0}}=\left[4\left(C_{j}(0)\right)^{2} \cdot \gamma_{j u}{ }^{2}(\omega) \cdot S_{u}(\omega)+\left(\frac{d C_{j}}{d i}(0)\right)^{2} \cdot \gamma_{j w}{ }^{2}(\omega) \cdot S_{w}(\omega)\right] \\
& +\left[2\left(C_{j}(0) \cdot \frac{d C_{j}}{d i}(0)\right) \cdot\left(\gamma_{j u w}{ }^{2}(\omega) \cdot S_{u w}(\omega)+\gamma_{j w u}{ }^{2}(\omega) \cdot S_{w u}(\omega)\right)\right]
\end{aligned}
$$

où $S_{u}(\omega)$ et $S_{w}(\omega)$ sont respectivement les fonctions de densité spectrale de puissance des fluctuations horizontale et verticale de la vitesse du vent ; $S_{u w}(\omega)$ est la densité interspectrale entre les composantes $u$ et $w$ de la turbulence $\gamma_{j u}(\omega), \gamma_{j w}(\omega), \gamma_{j u w}(\omega), \gamma_{j w u}(\omega)$ sont les fonctions d'admittance aérodynamique de la section, définies par $\gamma_{j l}{ }^{2}(\omega)=\Psi_{j l}(\omega)$. $\Psi_{j m}{ }^{*}(\omega),(j=x, z, M, l=u, w, m=u, w)$. «* " dénote l'opérateur de conjugaison. $S_{j}^{0}$ est un facteur de normalisation qui vaut $\frac{1}{4} \rho^{2} B^{2} U_{r e f}^{2}$ pour $j=x, z$, et le moment de référence $\frac{1}{4} \rho^{2} B^{4} U_{r e f}^{2}$ pour $j=M$. Les fonctions d'admittance aérodyna$\frac{1}{4}$

mique sont analogues à la fonction de Sears pour les ailes d'avion. Pour les corps mal profilés, il n'existe pas à l'heure actuelle de formulations analytiques pour ces dernières. Néanmoins, pour les tabliers de pont, quelques expressions semi-empiriques, inspirées de la formulation de Sears, ont été proposées pour approcher les fonctions d'admittance aérodynamique [10].

\subsection{Amortissement et rigidité aérodynamique}

Que ce soit dans une approche quasi-stationnaire ou aéroélastique, les vibrations induites par le vent sur un pont souple, discrétisé par éléments finis, conduisent à un système différentiel du second ordre :

$$
M \ddot{d}(t)+\boldsymbol{C} \dot{d}(t)+K \boldsymbol{d}(t)=F^{a e}\left(U_{r e f}, \dot{d}, d, t\right)+F^{t}\left(U_{r e f}, V^{\prime}, t\right)
$$

dans lequel $\boldsymbol{M}, \boldsymbol{C}$ et $\boldsymbol{K}$ sont respectivement les matrices de masse, d'amortissement et de rigidité de la structure (de taille $n \times n)$; $\mathrm{n}$ est le nombre de dégrés de liberté du système ; $d(t)$ est le vecteur de déplacements nodaux, $\boldsymbol{F}^{\mathrm{ae}}\left(U_{r e f}, \dot{\boldsymbol{d}}, \boldsymbol{d}, t\right)$ est le vecteur des efforts auto-entretenus, fonction de la vitesse moyenne du vent, des déplacements et des vitesses d'oscillation de la structure ; $\boldsymbol{F}^{t}\left(U_{r e f}, \boldsymbol{V}^{\prime}, t\right)$ représente le vecteur des efforts générés par le vent turbulent. Compte tenu des équations (6) et (8), il est possible de montrer que l'équation (14) devient dans le domaine fréquentiel :

$$
\begin{aligned}
& \left( \pm \omega^{2} \boldsymbol{M}+i \omega \boldsymbol{C}+\boldsymbol{K}\right) d(\omega)= \\
& =\overbrace{i \omega A\left(U_{r e f}, \omega\right)+\boldsymbol{B}\left(U_{r e f}, \omega\right) d(\omega)}^{\text {auto-entretenu }}+\overbrace{\boldsymbol{D ( \omega ) \boldsymbol { V } ^ { \prime } ( \omega )}}^{\text {turbulence }}
\end{aligned}
$$

Les matrices $\mathbf{A}\left(U_{r e f}, \omega\right)$ et $\mathbf{B}\left(U_{r e f}, \omega\right)$ et sont des matrices d'amortissement et de rigidité aérodynamique. Dans le cas de l'approche quasi-stationnaire, elles ne dépendent que de la vitesse. Elles sont donc à coefficients constants alors que dans l'approche aéroélastique, elles sont fonction de la fréquence.

Dans la plupart des codes de calcul utilisés par les bureaux d'études en France, l'approche quasi-stationnaire est appliquée avec deux simplifications supplémentaires: l'élimination des termes de couplage et la suppression du terme en $\dot{\alpha}$ faute de connaitre avec précision le point d'action des forces (qui d'ailleurs varie avec le temps). Seuls les termes correspondant à chaque degré de liberté sont conservés, ce qui conduit à définir des amortissements aérodynamiques pour le pompage et le balancement, et aucun pour le tangage. L'absence d'amortissement pour le tangage est généralement compensée en réintégrant le terme de Scanlan $A_{2}{ }^{*}$ pris à la fréquence de torsion correspondante. Une telle approche aboutit en général à une surestimation des effets du vent, et à l'absence de décalage fréquentiel puisqu'aucune rigidité aérodynamique n'est prise en compte. Le recours à une approche aéroélastique linéarisée reste donc le meilleur moyen de représenter le comportement au vent d'un pont comme un véritable système couplé structure-écoulement. Il est possible d'observer le rôle d'amortisseur joué par les effets aéroélastiques sur la réponse en calculant le rapport : 


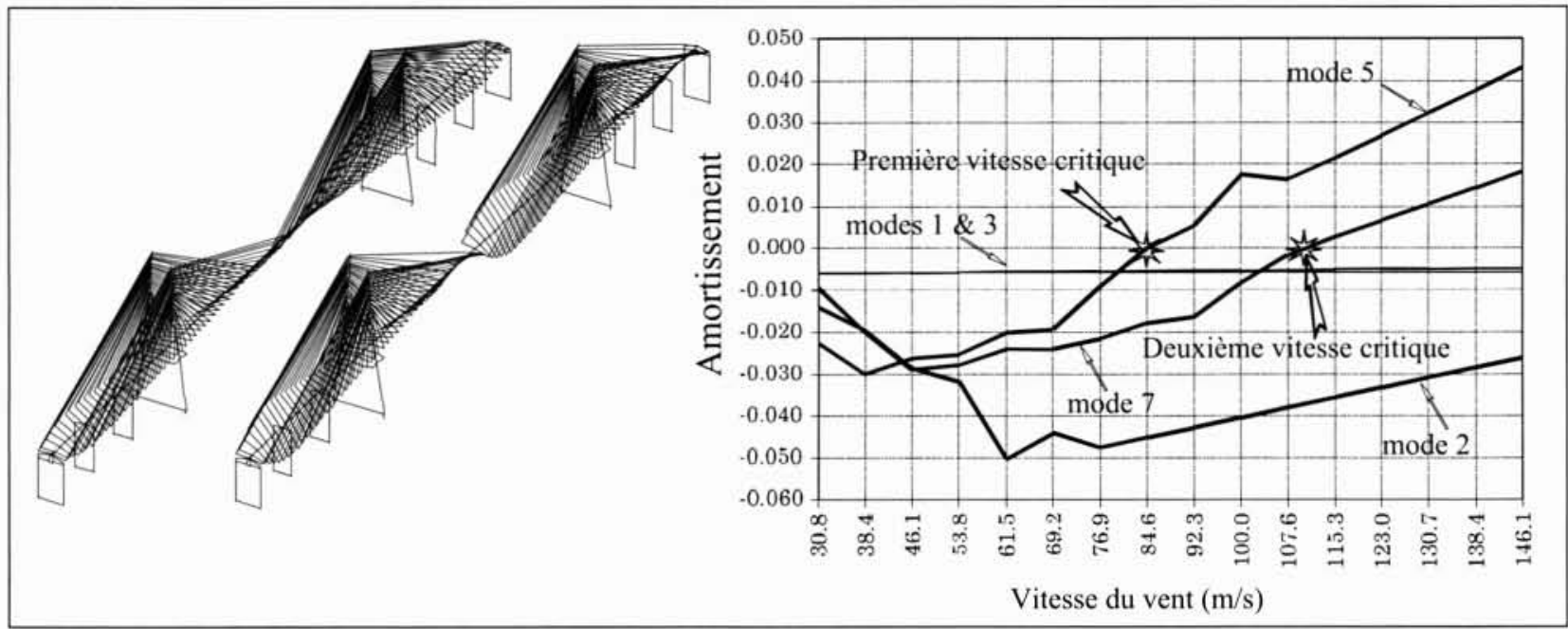

10 Calcul de la tenue au vent de la stabilité du pont Vasco de Gama.

$$
\eta=\frac{\max \left(S_{q}(\omega)_{\text {Pas de forces aero. }}\right)}{\max \left(S_{q}(\omega)_{\text {Avec de forces aero. }}\right)}
$$

(où $S_{q}(\omega)$ est la densité spectrale de puissance du mode) entre les valeurs aux pics de résonance de la réponse de deux calculs. Il vaut 57 pour le mode de torsion de la maquette du pont de Normandie. Ce même rapport est égal à $1 I$ pour le mode en torsion de la maquette du pont Vasco de Gama. Ces résultats confirment la bonne performance aérodynamique de la section profilée du pont de Normandie, par rapport à une section de type bi-poutre comme celle du pont Vasco de Gama.

Elle oblige cependant à manipuler des matrices d'amortissement et de rigidité dépendantes de la fréquence.

\section{- 3.5 Stabilité aéroélastique}

Le système couplé "fluide/structure " représenté par l'équation (12) devient instable lorsque, pour une vitesse de vent donnée, il existe une pulsation w qui annule le déterminant de la matrice d'impédance $G(\omega)$ :

$$
G(\omega)=\left[ \pm \omega^{2} \hat{\boldsymbol{M}}+i \omega\left(\hat{\boldsymbol{C}} \pm \hat{A}\left(U_{r e f}, \omega\right)\right)+\left(\hat{\boldsymbol{K}} \pm \hat{\boldsymbol{B}}\left(U_{r e f} \omega\right)\right)\right]
$$

Les matrices $\hat{\boldsymbol{M}}$ et $\hat{\boldsymbol{K}}$ sont les matrices diagonales de masse et de rigidité généralisées ; $\hat{\boldsymbol{C}}$ est la matrice d'amortissement généralisée qui n'est pas nécessairement diagonale ; $\hat{\boldsymbol{A}}\left(U_{r e f}, \omega\right)$ et $\hat{\boldsymbol{B}}\left(U_{r e f} \omega\right)$ sont les matrices d'amortissement et de rigidité aéroélastiques généralisées.

Toutes ces matrices sont obtenues après projection sur une base modale afin de réduire les dimensions du problème. La troncature modale est choisie pour une plage de fréquence représentant $90 \%$ de l'énergie du vent. Les vitesses du vent correspondant à l'annulation du déterminant de $G(\omega)$ sont appelées vitesses critiques de flottement (fig. 10). Une méthode de calcul possible est la méthode $\mathrm{pK}-\mathrm{F}$, méthode de type «point fixe $"[10]$.

\section{- 3.6 Réponse à la turbulence}

\subsubsection{Calcul spectral}

Les premières applications de la théorie des vibrations aléatoires à l'étude des structures soumises au vent turbulent sont dues à Liepmann (1952), adaptée plus tard pour l'ana- lyse des ponts suspendus par Davenport (1962). Cette méthode considère les forces produites par le vent turbulent sur la structure comme une action aléatoire (caractérisée par sa fonction densité spectrale de puissance) qui met en vibration la structure conformément à un phénomène résonance. Cette approche repose sur l'hypothèse de quasi-stationnarité et néglige tous les termes de couplage. Il est alors possible d'évaluer l'écart-type de l'amplitude des mouvements vibratoires correspondant à chaque mode propre de la structure.

Si les matrices d'amortissement et de rigidité aérodynamique définies dans l'équation (14) sont conservées, le calcul est moins aisé et oblige, pour chaque fréquence, l'inversion de la matrice d'impédance (il faut souligner qu'il n'est pas possible de recourir au concept de modes propres complexes dans ce cas car les matrices d'amortissement et de rigidité ne sont plus à coefficients constants). La densité spectrale de puissance de la coordonnée généralisée s'obtient alors par l'équation suivante :

$$
S_{q q}(\omega)=\boldsymbol{H}(\omega)^{\prime} \Theta \boldsymbol{D}(\omega) \boldsymbol{S}_{V^{\prime} V^{\prime}}(\omega)^{\prime} \boldsymbol{D}(\omega)^{*} \Theta^{\prime} \boldsymbol{H}(\omega)^{*}
$$

dans laquelle $S_{g q}(\omega)$ est la matrice de densités spectrales des coordonnées gênéralisées et $S_{V^{\prime} V^{*}}(\omega)$ la matrice de densité interspectrale de puissance des fluctuations. $\Phi$ est la matrice des modes propres (projection modale de réduction) et $H(\omega)$ est la matrice de transfert inverse de la matrice $G(\omega)$. L'écart-type d'une coordonnée généralisée s'obtient par inté-

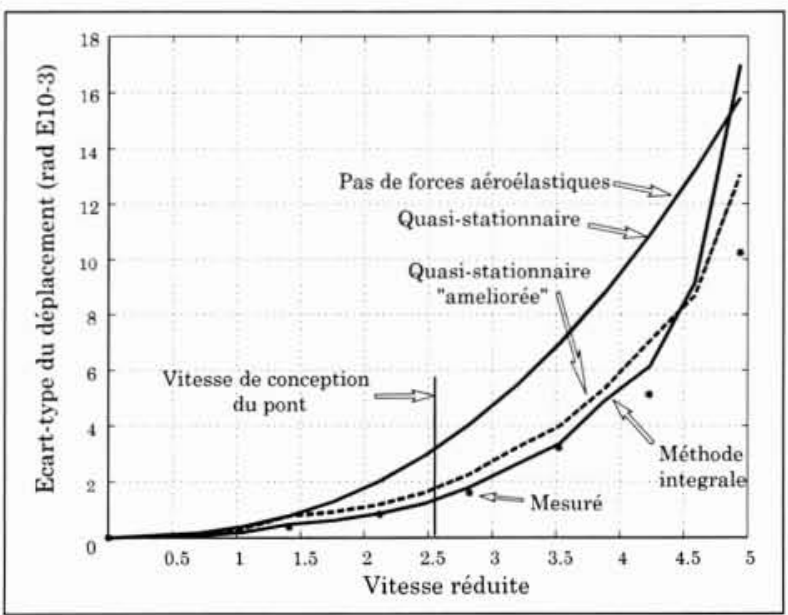

11. Evolution de l'écart-type de la rotation en torsion (maquette du pont de Vasco de Gama). 
gration de la densité spectrale. La densité spectrale d'un déplacement nodal s'obtient par combinaison des densités spectrales des modes, les densités interspectrales étant la plupart du temps négligées. La figure 11 donne une comparaison entre résultats issus d'une démarche quasi-stationnaire avec suppression des termes de couplage, et une approche totalement aéroélastique. Les résultats sont comparés avec ceux obtenus sur maquette à fils tendus.

\subsubsection{Calcul temporel}

Une approche alternative aux méthodes spectrales consiste à simuler des signaux aléatoires de la vitesse turbulente du vent aux nœuds de la structure puis à intégrer temporellement les équations de mouvement du système (fig. 12). Cela permet notamment de tenir compte de non linéarités géométriques ou matérielles. L'intégration temporelle des équations du mouvement peut être réalisée de façon approchée, en utilisant la méthode de Newmark. Pour les paramètres $\alpha=1 / 4$ et $\delta=1 / 2$, cet algorithme est inconditionnellement stable, c'est-à-dire qu'il existe une solution pour n'importe quelle valeur de $\Delta t$. Néanmoins, la précision des résultats obtenus n'est pas garantie. Deux phénomènes numériques se manifestent lors de l'utilisation des méthodes d'intégration directe (comme c'est le cas de la méthode de Newmark) : un amortissement numérique et un décalage fréquentiel. Le schéma d'intégration de Newmark avec accélération trapézoïdale est dit non dissipatif, c'est-à-dire dépourvu d'amortissement numérique [17]. Par contre le décalage fréquentiel peut induire des erreurs importantes dans l'estimation de la réponse. L'importance de ce phénomène est directement liée au pas de temps utilisé. Pour le minimiser, plusieurs auteurs [17] suggèrent d'utiliser la règle suivante :

$$
\frac{\Delta t}{T_{m}} \leq \frac{1}{10}
$$

où $T_{m}$ est la période d'oscillation du dernier mode de vibration $m$ qui contribue de manière importante à la réponse. La partie de la réponse, associée aux autres modes de vibration d'ordre supérieur, n'est pas évaluée correctement puisque la règle (16) n'est pas respectée. Ces effets parasistes peuvent être complètement éliminés en considérant uniquement les premiers $m$ modes de vibration par projection modale comme pour l'analyse spectrale. Enfin, le calcul temporel des effets du vent nécessite d'approximer les forces aéroélastiques. En effet, la formulation par fonctions indicielles est difficilement manipulable dans un schéma d'intégration. Le calcul temporel nécessite également de réaliser plusieurs simulations afin d'estimer les statistiques du processus de réponse (fig. 13).

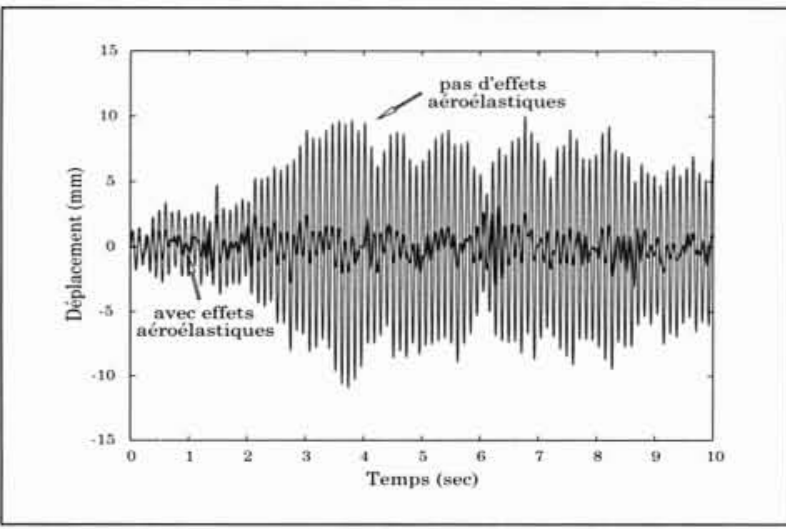

12 Réponse temporelle du déplacement vertical du modèle à fils tendus du pont de Normandie.

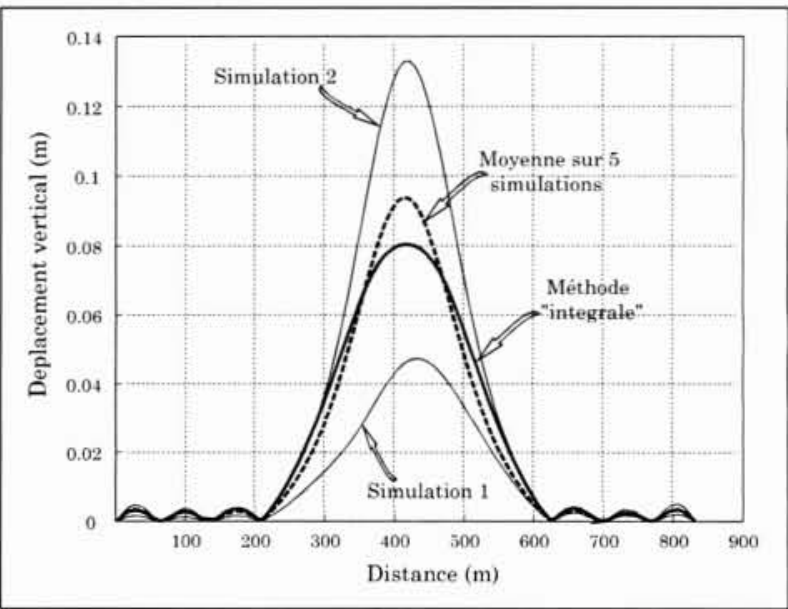

13 Ecart-type des déplacements verticaux (temporel/ spectral - maquette du pont vasco de Gama).

\section{IV — QUELQUES PROBLÈMES PARTICU- LIERS}

\subsection{Divergence en torsion}

Cette instabilité n'est pas de nature dynamique mais statique. Le phénomène de divergence en torsion a été mis en évidence sur les ailes de certains avions susceptibles de vriller à des vitesses d'écoulement d'air élevées. Ce phénomène peut se produire pour des tabliers de pont. Lorsque la vitesse du vent croît, le moment de torsion augmente, faisant vriller d'autant plus la section. L'angle d'attaque du vent change donc, accroissant ainsi le moment de torsion. L'ensemble du phénomène nécessite de la part de la structure un moment résistant additionnel. Finalement, une vitesse critique est atteinte dès que la demande additionnelle de moment résistant ne peut plus être satisfaite. Cela crée une condition d'instabilité qui mène la structure à rupture. Ce phénomène est à rapprocher de celui du flambement. La divergence en torsion ne dépend pas de la résistance ultime de la structure, mais de la flexibilité structurelle et de la manière dont le moment aérodynamique croît. Si le tablier est rectiligne et horizontal avec des caractéristiques aérodynamiques constantes sur toute sa longueur, en considérant que le pont subit seulement des oscillations en torsion, l'équation du mouvement du système :

$$
m_{\alpha} \ddot{\alpha}+c_{\alpha} \dot{\alpha}+k_{\alpha} \alpha=\frac{1}{2} \rho B^{2} U_{r e f}^{2} \cdot\left(K A_{2}^{*}(K) \frac{B}{U_{r e f}} \dot{\alpha}+K^{2} A_{3}^{*}(K) \alpha\right)
$$

Si la fréquence d'oscillation tend vers zéro, compte tenu du comportement asymptotique des coefficients instationnaires, l'équation (18) devient :

$$
k_{\alpha} \alpha=\frac{1}{2} \rho B^{2} U_{r e f}^{2} \frac{d C_{M}}{d i}(0) \alpha
$$

Il y a donc instabilité pour la vitesse critique :

$$
U_{\text {critique }}=\sqrt{\frac{2 k_{\alpha}}{\rho B^{2} \frac{d C_{M}}{d i}(0)}}
$$

Des phénomènes de divergence en flexion verticale et de divergence en balancement peuvent également être mis en évidence. A l'heure actuelle, aucune de ces instabilités n'a été constatée sur un ouvrage réel. 


\subsection{Galop}

Comme pour la divergence, le galop est un cas limite aéroélastique. En effet, il correspond à un coefficient d'amortissement total (aérodynamique et structural) qui s'annule. Le critère de Den Hartog qui l'exprime est établi suivant une démarche quasi-stationnaire. Son équivalent aéroélastique est par exemple le flottement à un degré de liberté en flexion verticale. En effet, si l'on considère seulement un mouvement de pompage :

$$
m_{h} \ddot{h}+c_{h} \dot{h}+k_{h} h=\frac{1}{2} \rho B U_{r e f}^{2} \cdot\left(K H_{1}^{*}(K) \frac{\dot{h}}{U_{r e f}}+K^{2} H_{4}^{*}(K) \frac{h}{B}\right)(20)
$$

le coefficient du terme en vitesse s'annule pour des couples de valeur $(\dot{\omega}, U)$ qui correspondent aux pulsations et aux vitesses critiques de flottement. Dans le cas où la fréquence réduite tend vers 0 , l'amortissement total devient au premier ordre :

$$
c_{h} \pm \frac{1}{2} \rho B U_{r e f} K H_{1}^{*}(K) \sim c_{h} \pm \frac{1}{2} \rho B U_{r e f} \frac{d C_{z}}{d i}(0)
$$

On retrouve bien la condition de Glauert-Den Hartog:

$$
U_{\text {critique }}=2 c_{h} / \rho B \frac{d C_{z}}{d i}(0) \text {. }
$$

\subsection{La mise en vibration des câbles et des suspentes de pont}

Les câbles ou les suspentes de grande longueur sont souven très souples et les nombres de Scruton $2 m \delta / \rho D^{2}$ ( $m$ étant la masse linéique, $\delta$ le décrément logarithmique, $D$ le diamètre du câble) sont généralement petits. De ce fait, des vibrations admettant de larges amplitudes peuvent être induites par le vent. A cela, s'ajoutent d'autres facteurs importants favorisant la mise en vibration : la présence d'autres câbles à proximité, de givre ou de filets d'eau à leur surface. Divers phénomènes peuvent donc apparaître : galop, galop de sillage, vibrations conjointes vent-pluie, échappements tourbillonnaires à nombre critique de Reynolds ou à haute vitesse,... Bien que l'on décrive parfois ces phénomènes suivant une démarche quasi-stationnaire, les mécanismes d'excitation sont encore très mal connus. La soufflerie est alors l'outil le mieux adapté à la compréhension du phénomène et à la définition de contre-mesures comme ce fut le cas pour le pont de Normandie [18].

Quel que soit le type de câble (à section circulaire ou trapézoïdale comme un ensemble de torons), la réponse peut être de type divergent. En fait, les travaux de Matsumoto [19] montrent qu'aux tourbillons de Von Karman viennent s'ajouter des tourbillons axiaux (c'est-à-dire dans l'axe du câble), amplifiant par là même les échappement tourbillonnaires.

Parmi ces phénomènes, l'excitation conjointe « vent/pluie " est celui qui a suscité le plus d'études. La présence de pluie semble maintenant être perçue comme un paramètre favorisant les instabilités. Ce phénomène fut rapporté pour la première fois sur le Meiko-Nishi Bridge, le Aratsu Bridge(Japon), le pont Dömitz (Allemagne), le pont Erasmus (Hollande). La fourchette des vitesses de vent est comprise entre 5 et $20 \mathrm{~m} / \mathrm{s}$, et le phénomène ne concerne que les câbles protégés par une gaine en polyéthylène. L'amplitude des vibrations peut atteindre plus de deux fois le diamètre du câble. Irwin [20] a proposé de choisir des nombres de Scruton supérieur à 10 pour éviter ces vibrations ventpluie.

L'échappement tourbillonnaire induit des vibrations de petites amplitudes, en général quelques millimètres. Si le phénomène a peu de chance de causer des problèmes sérieux, il se produit cependant pour des gammes de vitesse de vent assez larges, excitant des modes de vibrations de plus en plus élevés lorsque la vitesse augmente.

En général, l'augmentation du nombre de Scruton (réalisé par exemple en augmentant l'amortissement $\delta$ ) suffit pour dissiper les oscillations du câble. Mais, il convient d'insister sur le fait que des mesures prises pour éliminer un type de vibration peuvent s'avérer inefficaces pour réduire les oscillations dues à d'autres phénomènes.

\subsection{Echappements tourbillonnaires}

L'excitation par échappement tourbillonnaire peut être une cause importante de vibrations de ponts à haubans. Ainsi, le pont de St-Nazaire a été aérodynamiquement modifié par la mise en place de volets déflecteurs le long des arêtes du tablier, supprimant ainsi le phénomène. Avant modification de son profilé, la première vitesse de vent susceptible d'enclencher un phénomène d'échappement tourbillonnaire était très modérée. En effet, l'épaisseur du tablier étant de $3,40 \mathrm{~m}$, la fréquence fondamentale de flexion de $0,31 \mathrm{~Hz}$ pour un nombre de Strouhal de 0,11 , la vitesse était de $34 \mathrm{~km} / \mathrm{h}$. Pour le pont de Normandie, on trouve une première vitesse critique faible de $18,75 \mathrm{~km} / \mathrm{h}$. Cela a donc conduit à des études supplémentaires pour vérifier que les amplitudes maximales restaient acceptables. Pour une section de tablier, des nombres de Strouhal compris entre 0,1 et 0,4 sont réalistes.

Les vibrations de tabliers de pont dues aux échappements tourbillonnaires se produisent en général pour une gamme assez étroite de vitesses de vent ( 15 à $50 \mathrm{~km} / \mathrm{h})$. Les amplitudes maximales ne sont pas suffisamment importantes pour mettre en cause la pérennité de l'ouvrage, mais peuvent être inacceptables pour le confort de l'usager. Le changement de profil du tablier peut éliminer ce phénomène. Quelques vibrations peuvent cependant être acceptées, à condition que les accélérations ne dépassent pas $2 \%$ de l'accélération gravitationnelle pour des vitesses de vent inférieures à $50 \mathrm{~km} / \mathrm{h}$, et $5 \%$ pour des vitesses comprises entre $50 \mathrm{~km} / \mathrm{h}$ et $110 \mathrm{~km} / \mathrm{h}$.

\section{V — VERS LA SIMULATION NUMÉRIQUE « FLUIDE-STRUCTURE »}

Les études numériques présentées dans les paragraphes précédents reposent sur la connaissance de données aérodynamiques. Ces données sont fournies par les essais en soufflerie sur des tronçons d'éléments. Cette étape pourtant essentielle est consommatrice de temps mais également d'argent. Dans un budget d'études parfois serré, il n'est pas alors possible de tester toutes les variantes de sections souhaitées. La simulation numérique des écoulements (Computational Fluid Dynamics) est un domaine largement utilisé dans d'autres ingénieries concernées par les problèmes d'interaction fluide-structure. Ainsi, depuis quelques années, des tentatives ont été menées pour appliquer en premier chef ces outils à l'évaluation des caractéristiques aérodynamiques des ponts[12], [21].

\subsection{Simulation bi-dimensionnelle}

Comparée à la portée des ouvrages, la largeur des tabliers permet de traiter l'écoulement autour de chaque section comme un écoulement bi-dimensionnel. Cette hypothèse simplifie de beaucoup la simulation numérique. Cette approche est d'ailleurs celle employée dans les essais sur tronçons en soufflerie. L'analyse des fluides réels est consi- 


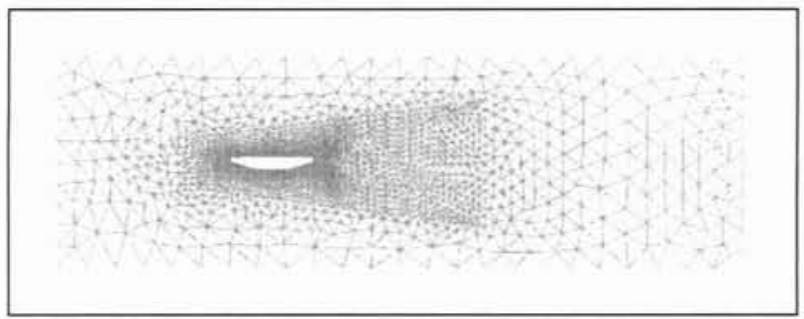

14. Maillage du fluide autour du tablier du pont de Millau (d'après [21]).

dérablement plus complexe que, par exemple, l'étude du comportement des structures en élasticité. Cela est dû à la viscosité qui introduit un élément d'incertitude dans l'écoulement et qui est responsable du développement de régions à forts gradients de cisaillement comme dans les couches limites et les zones de sillage. Dans le cas des écoulements autour des tabliers de pont, les couches limites développent des domaines régis par des lignes de séparation et des zones de recirculations variables dans le temps. Les simulations numériques doivent donc être capables de décrire ces changements. Elles doivent également couvrir des périodes de temps typiquement supérieures aux périodes d'oscillation de la structure.

Ce sont les équations de Navier-Stokes qui décrivent le mouvement d'un fluide :

$$
\begin{aligned}
& \nabla \boldsymbol{u}=0 \\
& \rho \partial_{t} u+\rho(u \cdot \nabla) \boldsymbol{u}= \pm \nabla p+\mu \nabla^{2} \boldsymbol{u}
\end{aligned}
$$

La première est l'équation de continuité et la seconde celle de la quantité de mouvement. Ce système doit être naturellement muni de conditions limites, notamment sur les parois solides, et initiales en temps.

Les équations de Navier-Stokes ne fournissent de solutions analytiques que pour quelques cas, souvent de portées limitées. En pratique, les équations sont discrétisées en temps et en espace. Dans les méthodes à discrétisation spatiale, un maillage est distribué sur la totalité du domaine où l'écoulement est étudié (fig. 13). Les vitesses et pressions sont calculées en tout point de maillage en vérifiant les conditions aux limites (fig. 14). Afin de correctement décrire les couches limites et les effets de sillage, ces maillages doivent être suffisamment fins. Ces méthodes fournissent également des algorithmes (méthodes $k-\varepsilon \ldots$...) qui permettent l'ajout de modèles de turbulence. Des techniques particulières (Arbitrary Lagragian-Eulerian formulation) facilitent l'étude de l'écoulement dans le cas où l'obstacle est en mouvement. Les travaux menés au LCPC, bien que prometteurs [21] restent encore à améliorer.

Cependant, dans le désavantage de ces méthodes réside l'effort nécessaire pour discrétiser le domaine d'étude de l'écoulement [12]. Cela peut paraître d'un intérêt limité lorsque seuls les coefficients aérodynamiques sont recherchés, requérant la seule connaissance des champs de pressions à la frontière de l'obstacle.

Le problème du maillage du domaine fluide peut être évité par des méthodes de discrétisation particulaire, une technique développée dans l'aéronautique dans les années 60 . La discrétisation est réalisée en divisant le contour de l'obstacle en petits éléments, chacun générant un tourbillon. Ces tourbillons élémentaires sont les solutions de l'équation des tourbillons, une reformulation des équations de Navier-Stokes :

$$
\begin{aligned}
& \nabla \times u=\omega \\
& \partial_{t} \omega+(u \cdot \nabla) \omega=v \nabla^{2} \omega
\end{aligned}
$$

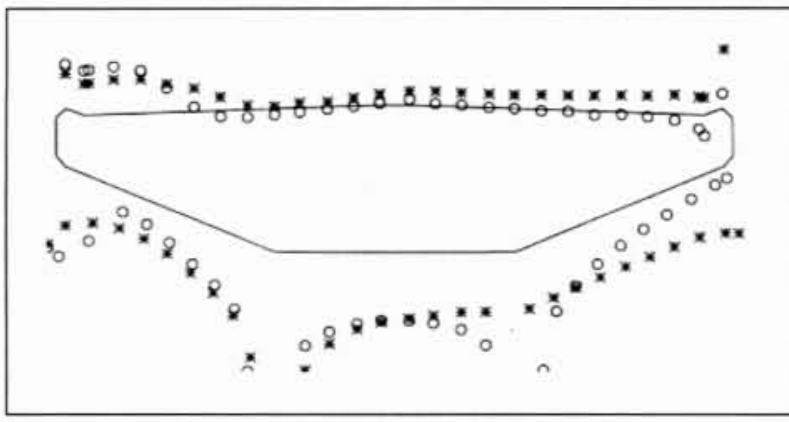

15. Champs de pression expérimentaux $\left({ }^{\circ}\right)$ et numériques (*) autour du tablier du pont de Millau (d'après [21]).

La première équation de (23) est remplacée par une relation de type Biot et Savart, permettant d'exprimer la vitesse en fonction du tourbillon (vortex). Cette relation est introduite dans la seconde équation. La résolution de ce problème s'effectue en plusieurs étapes : des tourbillons sont relâchés des éléments du contour de l'obstacle et sont diffusés par l'écoulement. De nouveaux tourbillons sont de nouveau formés à la surface de l'obstacle interagissant avec l'écoulement, les autres tourbillons et le déplacement éventuel des parois de l'obstacle. Ce processus permet de créer de larges structures cohérentes au sein de l'écoulement. Les pressions à la surface du corps ainsi que les efforts de portance, traînée et de torsion s'obtiennent alors directement [12].

\section{- 5.2 Simulation intégrale}

Le projet FSI-SD (Fluid Structure Interaction-Structural Design, $\mathrm{N}^{\circ}$ 20111), co-financé par l'Union Européenne, a pour ambition de développer une passerelle (FSI-Coupler) entre un code de calcul de structures et deux codes de calcul d'écoulements (compressible et incompressible) pour résoudre divers problèmes d'interaction fluide-structure en génie civil et maritime. Suivant la nature de l'écoulement (compressible ou non), le module de couplage différera. Les méthodes numériques sont également différentes entre le calcul structure et le calcul fluide. Ces problèmes de modélisation ne doivent pas avoir d'impact sur les calculs : le comportement de la structure détermine les conditions aux limites pour l'écoulement, et les variations de ce dernier modifient les forces s'exerçant sur la structure.

A chaque itération, le code fluide a besoin de connaître les nouvelles coordonnées du maillage déformé. Cela lui permet de calculer les caractéristiques de l'écoulement et les forces résultantes sur la structure. Le coupleur transfère dans le format adéquat les forces pour le code structure [22]. Les premiers calculs réalisés sur le Great Belt Bridge fournissent des vitesses d'instabilité avec moins de $10 \%$ d'erreurs par rapport aux essais en soufflerie.

Karniadakis et Newman [23] ont également réalisé des simulations sur le comportement vibratoire d'un câble dans un écoulement. En particulier, ils étudient les conditions à l'origine du lock-in. Le sillage est analysé sur des longueurs allant jusqu'à 10 fois le diamètre et pour des Reynolds compris entre 100 et 3900 . Le sillage en zone proche ( 3 fois le diamètre) est dominé la couche limite et est très sensible aux perturbations. Leur étude a porté sur plus de 20 simulations tri-dimensionnelles allant de 200000 à 100000000 degrés de liberté. Ces travaux se sont concentrés sur la dynamique de la couche limite et ses effets sur les vitesses de l'écoulement en zone proche de sillage, sur l'effet de la viscosité sur l'écoulement (air, eau). 


\section{VI $\square$ CONCLUSIONS}

David B. Steiman, le célèbre concepteur américain, définissait l'ingénieur comme... un artiste, et un poète aussi bien qu'un mathématicien, un scientifique, un financier et un entrepreneur. Avec les challenges que constituent les ponts de plus de 3000 mètres de portée, il faudra y ajouter une autre profession - celle d'aérodynamicien.

\section{RÉFÉRENCES}

[1] Faquharson, F.B. (1949-1954), "Aerodynamic stability of suspension bridges", University of Washington, Bulletin 16, part I-IV.

[2] DavenPort, A. G. (1972), "The use of taut-strip models in the prediction of the response of long span bridges to turbulent flow", Proc. Symp. On Flow-Induced structural Vibrations, IUTAM-IAHR, Karlsruhe, pp. 373-381.

[3] BLEICH, F. (1948), "Dynamic instability of truss-stiffened suspension bridges under wind action", Proc. ASCE, Vol 74. $\mathrm{N}^{*} 8$, pp. 1269-1314.

[4] RoCARD, Y. (1954), "L'instabilité en mécanique", Masson, Paris.

[5] THEODORSEN, T. (1934), "General theory of aerodynamic instability and the mechanism of flutter", National Aeronautics and Space Administration, NACA-report 496.

[6] Scanlan, R. H., Tomko, J.J. (1971), "Airfoil and bridge flutter derivatives", Journal of Engineering Mechanics, ASCE, Vol 109, $\mathrm{N}^{\circ} 2$, pp. 586-603.

[7] Davenport, A. G. (1962), "The buffeting response of a suspension bridge by storm winds", Journal of the Structural Division, ASCE, 88(33), pp. 233-268.

[8] Scanlan, R. H. (1978), "The action of flexible bridges under wind, I \& II", Journal of Sound and Vibration, Vol. 160, $\mathrm{N}^{\circ} 2$, pp. 187-211

[9] JaIN, A., Jones, N.P., SCANLAN, R. H. (1996), "Coupled flutter and buffeting analysis of long-span bridges". Journal of Structural Engineering, ASCE, Vol 122, $\mathrm{N}^{*} 7$, pp. 717-725.
[10] Patron, A. (1998), "Modélisation numérique de la tenue au vent des ponts souples", Thèse ENPC.

[11] Arzoumanidis, S. G., Bieniek, M. P. (1985), "Finite element analysis of suspension bridges", Computers \& Structures, Vol 21, $\mathrm{N}^{\circ} 6$, pp. 1237-1253.

[12] LARSEN, A., WALTHER, J.H. (1996), "Aeroelastic analysis of bridge girder sections based on discrete vortex simulations", Second International Symp. On computational wind Engineering, Fort Collins, Colorado.

[13] LCPC (1998-2001), "Effets du vent sur les structures de génie civil," Programme de Recherche LCPC-INRIA-CSTBENPC.

[14] Dowell, E. H. (Ed.), Crawley, E. F., Curtiss, H. C., Peters, D. A., Scanlan, R. H., Sisto, F. (1995), “A Modern Course in Aeroelasticity". Ed. Kluwer, U.S.A.

[15] SCanlan, R. H. (1993), "Problematics in formulation of wind-force models for bridge decks", Journal of Engineering Mechanics, ASCE, Vol 119, N 7, pp. 1353-1375.

[16] SCANLAN, R. H. (1987), "Interpreting aeroelastic models of cable-stayed bridges." Journal of Engineering Mechanics, ASCE, Vol 113, $\mathrm{N}^{\circ} 4$, pp. 555-573.

[17] Bathe, K. J. (1982), "Finite element procedures in engineering analysis," Prentice-Hall Inc., New Jersey, USA.

[18] Flamand, O. (1994), "Rain-wind induced vibration of cables," Proceeding of the International Conference on Cablestayed Bridges, Deauville, Vol. 2, pp. 523-531.

[19] Matsumoto M. (1999), "Wind induced vibration control of inclined cable," $3^{\text {rd }}$ Cable dynamics Symposium, Trondheim, pp. 123-132.

[20] IRwIN, P.A. (1997), "Wind vibrations of cables on cablestayed bridges," Proc. Of structure Congress XV, SEI/ASCE, Portland, Oregon, pp. 383-387

[21] Amandolese, X. (1999), "Validation expérimentale du code de simulation NSI3-IFS," Rapport interne LCPC.

[22] KWAMDAL, T. \& al. (1999), "Coupled simulation of vortex induced vibration of slender structures as suspension bridges and offshore risers", $3^{\text {rd }}$ Cable dynamics Symposium, Trondheim, pp. 117-122

[23] Karnadiakis, G.E. (1999), "Parallel Simulations of the Turbulent Near-Wake Dynamics of Rigid and Flexible Circular Cylinders", Department of Energy, http ://www.cfm.brown.edu/crunch/topics.html 